\title{
A Case of Sebaceous Carcinoma Mimicking a Giant Pyogenic Granuloma
}

\author{
Hyeree Kim, Hei Sung Kim, Sang Hyun Cho, Jeong Deuk Lee* \\ Department of Dermatology, Incheon St. Mary's Hospital, College of Medicine, The Catholic University of Korea, Seoul, Korea
}

Received: June 15, 2016; Accepted: August 19, 2016; Published: September 14, 2016

*Corresponding author: Jeong Deuk Lee, Professsor, Department of Dermatology, Incheon St. Mary's Hospital, College of Medicine, The Catholic University of Korea, 56 Donsuro, Bupyeong-gu, Incheon, 403-720, Korea. Tel: 82-32-820-5100; Fax: 82-2-506-9514; E-mail: leejd@olmh.cuk.ac.kr

Keywords: Sebaceous carcinoma Mimicking Granuloma Pyogenicum; sebaceous carcinoma on the arm; extraocular sebaceous carcinoma; Sebaceous carcinoma

Sebaceous carcinoma (SC) is uncommon disease that is often misdiagnosed as a benign lesion, but has an aggressive clinical behavior with a rate of distant metastasis of nearly $60 \%$ [1]. There are two main variants on the basis of their location; ocular and extraocular SC. Extraocular SC accounts for only about 25\% of all SC, and mostly involves the head and neck region, although it may occur at any site that contains sebaceous glands, including the trunk, extremities, and the external genitalia [2, 3]. To our knowledge, SC on the extremities is very rare. Here in, we report a case of sebaceous carcinoma on the left arm of a Korean woman.

A 96-year-old woman presented with a painful, solitary, $3 \times 3 \mathrm{~cm}$-sized, well-demarcated, flat-topped, reddish, erosive mass on the left arm for 5 years (Fig. 1). The lesion was on the site where she strapped watch, and had been gradually grown. It tended to bleed easily.

Histopathology revealed epidermal necrosis and irregular, variable-sized, tumor nests in the dermis (Fig. 2A). The dermal epithelial tumor cell nests are composed of pleomorphic foamycytoplasmic cells as well as atypical cells with frequent mitosis (Fig. 2B).From immunochemical staining, consequently Epithelial Membrane Antigen(EMA) showed transmembrane positivity of the sebaceous cells.

A final diagnosis of SC was made. We referred her to the department of oncology, and the CT scan for systemic evaluation was done. The chest CT showed axillary lymph node metastasis. She refused colonoscopy for evaluating Muir-Torre syndrome because of her old age. The lesion was removed by doing palliative operation for bleeding control. She came to follow-up visits for a month. We advised her to make a visit when she feels uncomfortable.

$\mathrm{SC}$ is a malignant tumor derived from the adnexal epithelium of sebaceous glands. The clinical presentation of extraocular SC is non-specific, but often described as firm, yellow-pink nodules that grow slowly, and $30 \%$ of cases present a hemorrhagic surface. The morphology of these tumors is varied as basal cell cancer, squamous cell cancer, granuloma pyogenicum, or neuroendocrine cancer, etc [2]. This diversity may be explained by the common embryologic origin of the folliculo-sebaceousapocrine unit being recapitulated in their neoplasms, as well [2].

Extraocular SC is rare. To date, it has been reported in the following area: the external auditory canal, nose, oral mucosa, salivary glands, scalp, parotid, larynx and pharynx, extremities, palmoplantar lesion, breasts, lungs, anal margin, penis, vulva and cervix [3]. The pathogenesis of this disease is unclear, but it is associates with Muir-Torre syndrome, Human papillomavirus,

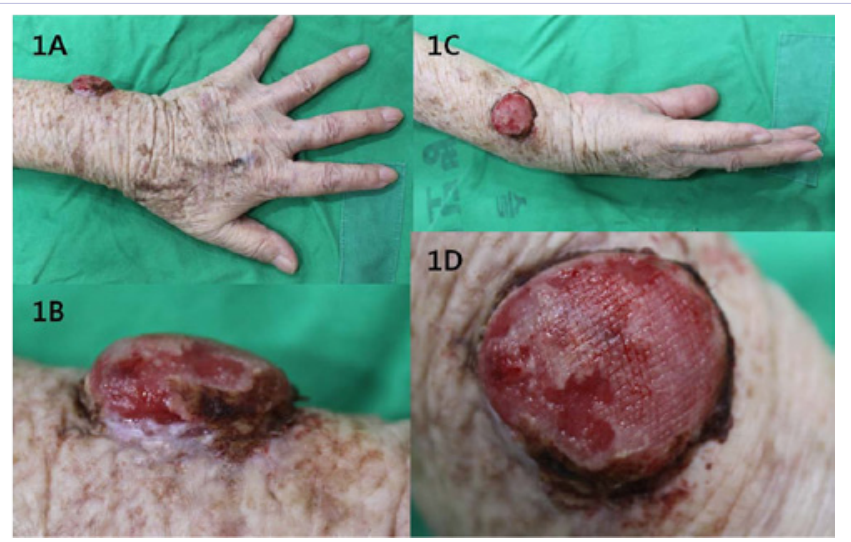

Figure 1: Clinical presentation: There is a solitary, $3 \times 3 \mathrm{~cm}$-sized, welldemarcated, flat-topped, reddish, erosive mass on the left arm.

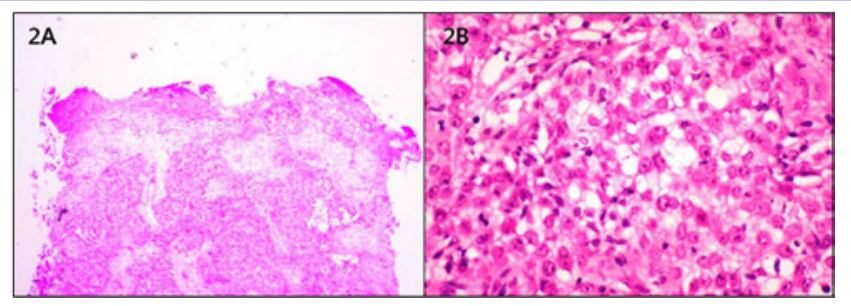

Figure 2: (A) The histopathology shows epidermal necrosis and irregular, variable-sized, tumor nests in the dermis. (H\&E, x40)

(B): The histopathology shows pleomorphic, vacuolated bubbly cytoplasm and atypical mitosis. (H\&E, x400) 
diuretics and radiotherapy. The gold standards of diagnosis is a biopsy of the lesion and surgery is the most efficient treatment, with margins of $5 \sim 6 \mathrm{~mm}$ [3]. Rao et al. suggested characteristics associated with poor prognosis:

Histopathologically vascular, lymphatic and orbital invasion; involvement of both eyelids; low differentiation; multicentric origin; over 6 months, over $10 \mathrm{~mm}$; pagetoid invasion and infiltrative pattern, etc [3].

To our knowledge, only 4 cases of SC have been reported on the arm $[1,2,4,5]$. This case was described for providing as rare case with arising on the left arm and resembling a giant pyogenic granuloma.

\section{References}

1. Piazza A, Gu X, Kim RH. Sebaceous carcinoma of the Rt. Arm. Journal of the Louisiana State Medical Society. 2012;164(2):81-82 .

2. Poonam KP, Rajalakshmi T, Julian AC, Suraj M, Pritilata R. Extraocular sebaceous carcinoma:a series of three cases with varied presentation. Dermatology Practical \& Conceptual. 2012;2(1):39-44. doi: 10.5826/ dpc.0201a07.

3. David OL, Fred BF, Hernando V, Bernard KK, Maria RP, Jose AC. Inguinal ulcerated sebaceous carcinoma: an unusual presentation. Anais Brasileiros de Dermatologica. 2013;88(6):48-51.

4. Phyu PA, Meenakshi B, Marjan M, Lynne J. Extraocular sebaceous carcinoma in situ: report of three cases and review of the literature. Journal of cutaneous pathology 2014;41(7):592-596. doi: 10.1111/ cup. 12351

5. Oka K, Katsumata M. Intraepidermal sebaceous carcinoma: case report. Dermatologica. 1990;180(3):181-185. 